\title{
Superstrong Field Science
}

\author{
T. Tajima* and G. Mourou ${ }^{\dagger}$ \\ ${ }^{*}$ Lawrence Livermore National Lab., Univ. of California, Livermore, CA 94550 \\ ${ }^{\dagger}$ Center for Ultrafast Optical Science, University of Michigan, Ann Arbor, MI 48109
}

\begin{abstract}
Over the past fifteen years we have seen a surge in our ability to produce high intensities, five to six orders of magnitude higher than was possible before. At these intensities, particles, electrons and protons, acquire kinetic energy in the mega-electron-volt range through interaction with intense laser fields. This opens a new age for the laser, the age of nonlinear relativistic optics coupling even with nuclear physics. We suggest a path to reach an extremely high-intensity level $10^{26-28} \mathrm{~W} / \mathrm{cm}^{2}$ in the coming decade, much beyond the current and near future intensity regime $10^{23} \mathrm{~W} / \mathrm{cm}^{2}$, taking advantage of the megajoule laser facilities. Such a laser at extreme high intensity could accelerate particles to frontiers of high energy, tera-electron-volt and peta-electronvolt, and would become a tool of fundamental physics encompassing particle physics, gravitational physics, nonlinear field theory, ultrahigh-pressure physics, astrophysics, and cosmology. Such a laser intensity may also be very beneficial to an alternative, more direct approach of fast ignition in laser fusion. We suggest a new possibility to explore this.
\end{abstract}

\section{INTRODUCTION}

Over the past fifteen years, we have seen a revolution in laser intensities [1]. This revolution stemmed from the technique of chirped pulse amplification (CPA), combined with recent progress in short-pulse generation and superior-energy-storage materials like Ti:sapphire, Nd:glass, and Yb:glass. The success of this technique was due to its general concept, which fits small, university-type, tabletop-size systems as well as large existing laser chains built for laser fusion in national laboratories like CEA-Limeil in France, Lawrence Livermore National Lab. (LLNL), Los Alamos National Lab., and Naval Research Lab. in the U.S.; Rutherford in the UK, Max Born Institute in Germany, and the Institute of Laser Engineering in Osaka, Japan. A record peak power of petawatt $\left(\mathrm{PW}=10^{15} \mathrm{~W}\right)$ has been produced at LLNL. CPA lasers have given access to a regime of intensities that was not accessible before, opening up a fundamentally new physical domain [2]. After a rapid increase in the 1960s with the invention of lasers, followed by the demonstration of $Q$-switching and mode-locking, the power of lasers stagnated due to the inability to amplify ultrashort pulses without causing unwanted nonlinear effects in the optical components. This difficulty was removed with the introduction of the technique of chirped pulse amplification, which took the power of tabletop lasers from the gigawatt to the terawatt - a jump of three to four orders of magnitude. This technique was first used with conventional laser amplifiers and more recently extended to Optical Parametric Chirped Pulse Amplifiers (OPCPA) [3]. A number of laboratories are presently equipped with CPA ultrashort-pulsed terawatt lasers such as Laboratoire Optique Applique in France, University of Lund in Sweden, Max-Planck Institute in 
Garching, Jena University, and the Japan Atomic Energy Research Institute in Kansai. The CPA-enabled short-pulse generation has advanced to the single-cycle regime [4]. The peak power in the 1990s reached 100 TW, demonstrated at JAERI Kansai. More recently, deformable mirrors have been incorporated into CPA, making it possible with low f\# parabola to focus the laser power on a $1 \mu \mathrm{m}$ spot size [5]. Present systems deliver focused intensities in the $10^{20} \mathrm{~W} / \mathrm{cm}^{2}$ range. In the near future, CPA systems will be able to produce intensities of the order of $10^{22} \mathrm{~W} / \mathrm{cm}^{2}$. As indicated in Fig. 1, we will see a leveling off of laser intensity for tabletop-size systems at $10^{23} \mathrm{~W} / \mathrm{cm}^{2}$. This limit [1] is imposed, as we will explain later, by the saturation fluence - energy per unit area of the amplifying medium and the damage threshold of the optical elements. In a wellconceived CPA system the saturation fluence is of the order but less than the damage threshold. Once this limit is reached, we will have accomplished a leap in intensities of eight orders of magnitude and the only way to increase the focused intensity further will be by increasing the beam size, leaving the following questions to be answered: How could we go higher in intensity? And how much higher?

Because the highest intensities will rely on the largest pump available, we explore if it could be technically feasible to build a large scale CPA (OPCPA) pumped by a megajoule system of the type of the NIF (National Ignition Facility) in the U.S. and the LMJ (Laser Megajoule) in France. Power in the zetta $\left(10^{21}\right)$ watt range could be produced, yielding a focused intensity of $10^{28} \mathrm{~W} / \mathrm{cm}^{2}$. These intensities well beyond the currently accessible will open up a new physical regime. The trend in intensity increase is represented in Fig. 1.

\section{En route to "zettawatt"}

Although a zettawatt system could be built using Yb:glass, with the advantages of being relatively compact due to the high $F_{\text {sat }}$ of this material and being diode pumpable, much development work needs to be accomplished to reach this intensity level with this material. The proposed systems described below have been stimulated by the construction, both in France and in the U.S, of lasers delivering a few megajoules of energy as well as the availability of large telescope technology (10m diameter) and deformable mirrors.

Let us recall that the NIF and LMJ systems will deliver $2 \mathrm{MJ}$ at $350 \mathrm{~nm}$, i.e., the third harmonic of $1060 \mathrm{~nm}$ in $3 \mathrm{~ns}$. The energy at $530 \mathrm{~nm}$, for a long pulse of 10$20 \mathrm{~ns}$, could be as high as $5 \mathrm{MJ}$. This energy could be used to pump a CPA-type system to produce of the order of $1 \mathrm{MJ}$ of energy in $10 \mathrm{fs}$ on a spot size of $1 \mu \mathrm{m}$ [5]. Such a system will have a power of the order $10^{20} \mathrm{~W}$ or 100 exawatts with an intensity of $10^{28} \mathrm{~W} / \mathrm{cm}^{2}$. To produce these phenomenal characteristics, we have two alternatives. The first one would be to use this large pump energy to drive an OPCPA system. This elegant technique [3] has been demonstrated to the joule level at $800 \mathrm{fs}$ level a few years ago by I. Ross et al. from Rutherford [6] and is being implemented in the same laboratory to the $10 \mathrm{PW}, 30 \mathrm{fs}$ level. It has the advantage that it could use KDP as the nonlinear medium. KDP has been produced in large dimensions at a relatively low cost for the $\mathrm{NIF}$ and LMJ programs. The working fluence on the crystal will be $1 \mathrm{~J} / \mathrm{cm}^{2}$, leading to a 


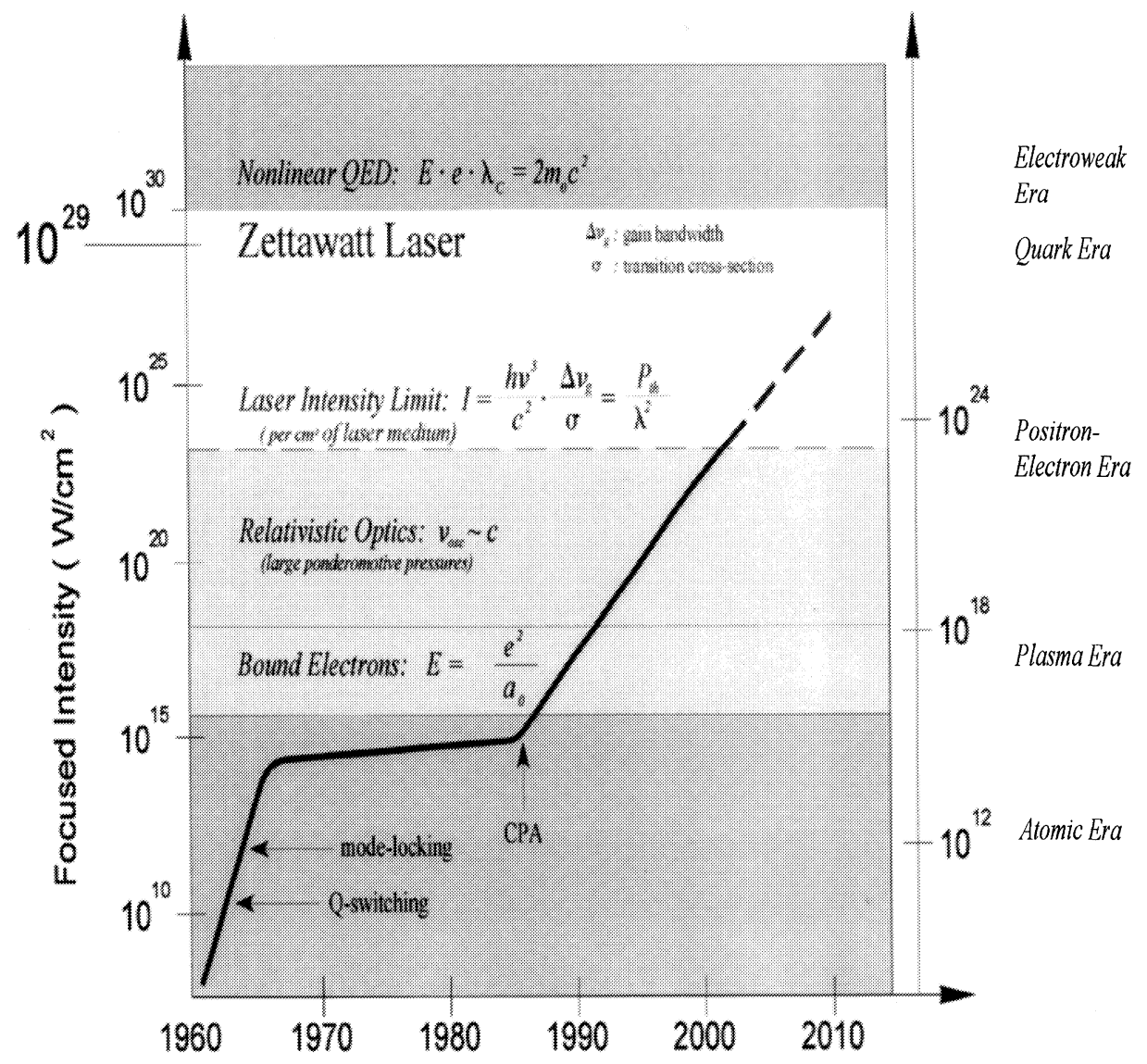

FIG. 1. The leap in laser intensity in time and new frontiers that access. 
beam diameter of around $10 \mathrm{~m}^{2}$ to accommodate the whole pump energy. This technique has the potential to produce a $10-\mathrm{fs}$ pulse or shorter, but needs to be demonstrated at least at the joule level. The second approach, though more conservative, will be to drive a CPA Ti:sapphire, a well-established technology. The amplifier will be composed of a large matrix of Ti:sapphire rods. The size of the matrix will be dictated by the saturation fluence $F_{\text {sat }}=1 \mathrm{~J} / \mathrm{cm}^{2}$, corresponding to a $10 \mathrm{~m}$ diameter beam. The amplifier could be composed of $2500,20 \times 20 \mathrm{~cm}$ pieces, each $2 \mathrm{~cm}$ in length. Each piece will have to be segmented in order to avoid the transverse amplified spontaneous emission. Ti:sapphire of $20 \times 20 \mathrm{~cm}$ has already been grown [10]. In both schemes the beams can be focused by a large parabola. The dielectric coating used for the parabola will have a damage threshold of $1 \mathrm{~J} / \mathrm{cm}^{2}$ (for short pulses) [7, 8], imposing a parabola size of $10 \mathrm{~m}$ in diameter. This parabola will have the same diameter as the Keck telescope. The phase front will be interferometrically controlled by an active matrix of deformable mirrors. The grating compressor could be made out of meter-size gratings assembled interferometrically in a matrix geometry. The size of the grating for a megajoule shortpulse system will be also of the order of $10 \mathrm{~m}$ in diameter, dictated by a damage threshold of $1 \mathrm{~J} / \mathrm{cm}^{2}$ (such a damage threshold has just been reported [9]). Each large grating will be composed of 100 of $1 \mathrm{~m}^{2}$-size gratings.

Note that although large, the number of optical components involved will be small, compared to a MJ system calling for 4500 laser slabs, 800 large KDP frequency converter crystals, and 500 gratings of meter-size. An alternative method using a plasma has been suggested [10], but will lead to the same output energy limited by the pump. In addition the beam must go through a plasma, which is highly undesirable.

\section{A Stepping stone: The exawatt laser system}

If a zettawatt laser, although feasible, could seem too grandiose at this time, an exawatt system on the other hand, which would produce $10 \mathrm{~kJ}$ in $10 \mathrm{fs}$, i.e., $10^{25} \mathrm{~W} / \mathrm{cm}^{2}$, could be readily constructed. Only one percent or $30 \mathrm{~kJ}$ of the NIF/LMJ energy would be necessary. The beam size will be of the order of one meter in diameter. The amplifying method will be composed of a matrix of 25 Ti:sapphire $20 \times 20 \mathrm{~cm}^{2}$ crystals [10] and two gratings of meter-size. The segmented telescope will have a one-meter aperture. The wave front will be corrected by a large deformable mirror.

In the following we explore a few examples of applications of such intense lasers that may enable new ways to investigate fundamental physics.

\section{HIGH-FIELD SCIENCE IN EXTREME FIELDS}

The major signpost of contemporary high-field science is the entry into the relativistic regime, which is characterized by the quivering momentum of electrons in the laser fields reaching the speed of light times the electron rest mass. This field is reached when the laser intensity is on the order of $10^{18} \mathrm{~W} / \mathrm{cm}^{2}$ for a typical optical frequency of the laser we mentioned above. When the laser field is much less than this, free electrons behave 
harmonically to the field's optical oscillations. Although these oscillations of electrons are still important enough to couple with various collective motions of free electrons, there is small orbital nonlinearity associated with the quivering motion in this regime. For bound electrons in atoms or molecules the quivering motion in the laser fields is once again, by and large, a perturbation on their orbital motion. This perturbation is enough to cause various interesting phenomena in the non-relativistic regime. There is such a wealth of phenomena known in this regime that it is hard to list them briefly, but they include the multi-photon process of ionization, that of transitions, Raman and Brillouin scatterings, and various optical nonlinearities [11] arising from the material's response. In the relativistic regime with the intensity above $10^{18} \mathrm{~W} / \mathrm{cm}^{2}$, in addition to the above phenomena, there emerge new classes of effects largely arising from the relativistic nonlinearities of electrons in the high field.

The field intensity in this regime means that the electron momentum in the light is typically $e E_{0} \omega_{0}$, which becomes of the order of and exceeds $m_{0} c$, where $\omega_{0}$ is the laser angular frequency, $E_{0}$ the laser electric field, and $m_{0}$ the electron rest mass. When the electron momentum exceeds $m_{0} c$, the electronic orbit ceases to be harmonic and linear. It becomes a figure- 8 motion, including higher-harmonic components. The photon pressure is exerted individually on an electron by the electron-photon collision through the Thomson cross-section

$$
\sigma_{T}=\frac{8 \pi}{3} \cdot \frac{e^{4}}{m_{0}^{2} c^{4}} \approx 7 \cdot 10^{-25} \mathrm{~cm}^{2} .
$$

When the flux of laser at the intensity entering the relativistic regime is shone on an electron (with Lorentz factor $\gamma$ ), this causes a force on it

$$
F=\frac{\sigma_{T}}{\gamma} \frac{E_{0}^{2}}{4 \pi} .
$$

When the laser intensity is $I=10^{26} \mathrm{~W} / \mathrm{cm}^{2}$, the force acting on an electron is $10^{-2} \sim$ $10^{-1} \mathrm{erg} / \mathrm{cm}$. Or the acceleration acting on an electron (originally) at rest is

$$
a_{e}=\frac{f}{m_{0}} \sim 10^{25-26} \mathrm{~cm} / \mathrm{s}^{2},
$$

where $m_{0}$ is the rest mass. [In contrast to this, the Schwinger acceleration is

$$
a_{S}=2 \cdot 10^{31} \mathrm{~cm} / \mathrm{s}^{2},
$$

at which an electron gains energy by $m_{0} c^{2}$ over the Compton length $\lambda_{C}=\hbar / m_{0} c$, and the pair creation becomes prevalent.] A large flux of photons bombarding an electron causes such high acceleration through collisions between photons and electrons (Compton collisions). Such acceleration may be called the Eddington acceleration, as Eddington introduced the stellar luminosity at which the gravitational pull is balanced by this photon collisional acceleration (the Eddington luminosity).

The Eddington acceleration may be likened to the acceleration of water molecules near the surface of water in a lake when there is a breeze passing over the water 
surface. The collisional viscosity created by collisions between water molecules and wind molecules gives rise to a water flow. However, we also observe that when the breeze gets stronger or becomes a gale, the water surface is no longer smooth and acquires ripples or waves. Such ripples facilitate an increase in the effective viscosity of water for the wind, so that the wind momentum is anomalously effectively transported to water molecules with a much faster rate through the Kelvin- Helmholtz instability at the interface of the two fluids. What happens in the case of strong photon flux ("wind") in a plasma? Just like the strong wind on the water surface, the strong photon flux is capable of creating plasma waves, which in turn causes enhanced viscosity and thus anomalous momentum transport from photons to electrons of the plasma. This latter process is through a collective interaction. The plasma wakefield excitation [12] is typical of this, in which plasma waves generated in this process are accentuated, and new processes of collective interaction emerge. In this ultraintense regime, electrons may be accelerated not only through the electrostatic field that is set up by the ponderomotive force of the laser, but also directly by the ponderomotive force itself to very high energies. If we apply this laser at the resonance absorption at high densities near the compressed laser fusion target, on the other hand, much of the laser energy may be converted into relatively low-energy copious electrons, which could constitute a new alternative to the fast ignition fusion. The acceleration of heavier particles (protons and other nuclei) to relativistic energies will become possible, too. Either by direct baryon acceleration by this, or other process, (it will take a variety of experimental realizations such as a target irradiation, cluster irradiation, converging imploding shells, etc.), we will access the nuclear regime of matter reminiscent of the early epoch of the Big Bang .

The production of extremely high energy or copious gamma rays will happened. The extreme high photon pressure (which already exceeds Gbar in the presently available intense lasers) may be finally directly utilized to directly compress matter in this ultrarelativistic regime, because baryons, too, become relativistic. If so, unprecedented densities of matter may be created. The combination of extreme intensity lasers and high-energy particle beams that can be created by the conventional high-energy physics accelerator will further multiply our ability to expand our frontier horizon. This will be the merging point of high-energy physics and high-field science. In the following, we list several examples of exciting new frontiers of fundamental physics that may be explored by this regime of intensities. In many of the applications we discuss, in this high-intensity regime the interaction length between the laser and matter is expected to be extended beyond the Rayleigh length, as the relativistic mass effect of electrons sets the self-focusing threshold at $10^{10}\left(\omega / \omega_{p}\right)^{2} \mathrm{~W}$ [13].

\section{Particle acceleration}

The pulse or self-modulation of a photon wavepacket with sufficient intensity induces a longitudinal electric field (in the $x$-direction, as part of plasma oscillations) as

$$
E_{x} \sim \sqrt{\frac{n_{e}}{n_{18}}} a_{0}^{2} \quad(\mathrm{GeV} / \mathrm{cm}), \quad\left(a_{0} \leq 1\right),
$$


where $n_{18}=10^{18} / \mathrm{cm}^{3}$ and $n_{e}$ is the electron density, $a_{0}=e E_{0} / m_{0} \omega_{0} c$ the normalized vector potential of the laser, which is sometimes called the quivering velocity normalized to $c$ (or quivering momentum normalized to $m_{0} c$ ). The energy gain over the interaction length $l_{x}$ is

$$
\Delta \varepsilon \sim \sqrt{\frac{n_{e}}{n_{18}}} a_{0}^{2} \ell_{x}(\mathrm{GeV}) .
$$

When $a_{0}$ exceeds unity (ultrarelativistic; $I \geq 10^{18} \mathrm{~W} / \mathrm{cm}^{2}$ ), the pressure of the photon wavepacket becomes so large that nearly all electrons are evacuated from the laser packet [14]. The photons plow through the plasma with electrons piling up in front of the pulse ("snow plow" of electrons), yielding the snow plow acceleration [15] with momentum gain of electrons

$$
p_{x}=\frac{1}{4} \frac{E_{0}^{2}}{n_{e} c}=\frac{E_{c}^{2}}{n_{e} m_{0} c^{2}} a_{0}^{2},
$$

where $E_{c}=m_{0} \omega_{0} c / e$. Here the laser pulse is assumed to have fully interacted with the plasma. If the pulse quickly diffracts before the full interaction, say over the Rayleigh length, $p_{x}$ is simply proportional to $E_{0}$.

Now let us imagine, for the moment, that the laser has only half the period (unipolar) [16] or subcyclic [17]. In this case the energy (or momentum) gain is

$$
\Delta \varepsilon \sim m_{0} c^{2} a_{0}^{2} .
$$

Since the Lawson-Woodward theorem [18] prohibits any overall acceleration for fully oscillatory (i.e., usual) electromagnetic waves in vacuum in infinite space, the above energy gain is compensated for by decelerating phase. There are, however, many instances that break the theorem requirements. For example, it may be possible in this extreme relativistic regime that electrons are accelerated to very high energy, immediately reaching the speed of light and becoming in phase with the photon over a sufficiently long distance, so that by the time they become dephased, the EM wave may decay away for some reason, such as by radiative decay or pump depletion to the acceleration. If this happens, the above energy gain (or a portion of it) may be preserved. In extreme high-intensity regimes such effects will become significant. If the laser spreads over the Rayleigh length, the energy gain is proportional to $a_{0}$. The transverse momentum gain $\left(p_{y}\right)$ is always proportional to $a_{0}$. In a broad general way we can say that the interaction between the laser and electrons becomes more coherent, as the laser intensity increases because the laser and electrons move more coherently over greater distance. This is the signature of the acceleration by the ponderomotive potential of photon fields in ultrarelativistic intensity regimes.

Thus it is possible to see electrons at energies of up to $\sim 100 \mathrm{TeV}$ at the laser intensity of $10^{26} \mathrm{~W} / \mathrm{cm}^{2}$ and even up to $\sim 10 \mathrm{PeV}$ at $10^{28} \mathrm{~W} / \mathrm{cm}^{2}$. The accelerating gradient is $200 \mathrm{TeV} / \mathrm{cm}$ and $2 \mathrm{PeV} / \mathrm{cm}$, respectively. Note that such energies $(100 \mathrm{TeV}$ and $10 \mathrm{PeV})$ if collided, correspond to $10^{19} \mathrm{eV}$ and $10^{23} \mathrm{eV}$ for fixed target experiments. These energies rival or exceed those of the highest energy cosmic rays, which are observed up to $3 \times 10^{20} \mathrm{eV}$. Of course, it is not easy to attain correspondingly high luminosity for collisions at such high energies. Even though the exploration of particle physics at pb 
may not be within reach, we may use such particles in pursuit of (other) fundamental physics at the energy frontier. We might recall that Anderson first discovered mesons in cosmic rays, followed by more detailed studies of those particles in cyclotrons and other accelerators. Perhaps the present way-out parameters in the energy frontier may herald some new phenomena. One such example may be the test of Lorentz invariance [19] in extreme high energies. For such a test, unlike the detection of new particles with $\mathrm{pb}$ cross-section, the luminosity requirement may be much relaxed.

When we irradiate an extremely relativistic laser pulse $\left(I \sim 10^{26} \mathrm{~W} / \mathrm{cm}^{2}\right)$ on a thin film ( $\gtrsim 1 \mu \mathrm{m}$ ) of a metal at a tight spot of $(1 \mu \mathrm{m})^{2}$ followed by a microhole in a metallic slab over more than one $\mathrm{cm}$, we hypothesize that the laser pulse picks up metallic electrons from the film and continues to propagate through the microhole, as it remains focused. If this proves to be the case, the amount of electrons that are to be accelerated by this pulse is in the ballpark of

$$
N_{e} \sim n_{e} A l_{b} \sim 3 \cdot 10^{10},
$$

where we assumed $n_{e} \sim 10^{24} \mathrm{~cm}^{-3}$, area $A=(1 \mu \mathrm{m})^{2}$, the bunch length $l_{b} \sim 3 \cdot 10^{-6} \mathrm{~cm}$. In such a large pickup, the pump depletion due to the energy transfer to electrons is significant. In fact, it may play a fundamental role in this acceleration to turn the electromagnetic energy into particle kinetic energy without returning to the decelerating phase, providing one way to break the Lawson-Woodward theorem's constraint, as we mentioned earlier. If we take $1 / 10$ of the above electrons to gain $10 \mathrm{TeV}$ and if we focus electrons (and positrons) down to $10^{-6} \mathrm{~cm}$ (or $10^{-7} \mathrm{~cm}$ ) at focus (the collision point), the luminosity of the colliding events is of the order of

$$
\mathcal{L}=10^{31} \mathrm{f} / \mathrm{cm}^{2} / \mathrm{s},\left(\text { or } 10^{33} \mathrm{f}\right),
$$

where $f$ is the collision repetition rate. Nakajima has considered a similar but more daring luminosity scenario [20].

Although we have no room to enumerate the x-ray generation from those high fields and subsequent high energy electrons, the quantity and quality of emitted $\mathrm{x}$-rays are extraordinary in this regime. This will be discussed again in the future.

\section{Fast ignition fusion}

One special case of laser energy conversion into electrons is through the resonance absorption at the critical density. The concept of fast ignition in laser-driven inertial fusion [21] calls for laser beam of $\sim 10$ psec duration at the intensity exceeding $10^{20} \mathrm{~W} / \mathrm{cm}^{2}$ to be absorbed at the critical density $\left(\sim 10^{21}-10^{22} / \mathrm{cm}^{3}\right)$, creating a beam of electrons in the several $\mathrm{MeV}$ range. The idea is to separate the roles of laser into two functions: one to compress the fuel with least amount of entropy increase so that the fusion fuel is compressed to a highest density with least amount of laser energy, and the other is to heat the fuel to the thermonuclear ignition temperature $(\sim 10 \mathrm{KeV})$ when the main compression is achieved. The latter step may be carried out according to Tabak

et al. by the appropriate range of energetic (several MeV) electrons that are transported from the crust of the target $\left(<10^{22} / \mathrm{cm}^{3}\right)$ to the surface of the fuel $\left(\right.$ at $\left.\sim 10^{26} / \mathrm{cm}^{3}\right)$ at 
the pulse duration of $\sim 10 \mathrm{ps}$. In order for electrons to trigger the fusion ignition, the condition

$$
\rho r \lesssim 0.5 \quad\left(\frac{\mathrm{g}}{\mathrm{cm}^{2}}\right)
$$

has to be fulfilled [21]. Here $\rho$ is the density of the compressed fuel and $r$ the electron range and thus approximately the size of the fuel at compression. The laser pulse length is therefore given as

$$
\tau=40\left(\frac{100 \mathrm{gcm}^{-3}}{\rho}\right) \mathrm{ps}
$$

which yields the pulse length between $10-20$ ps for the compressed fuel density of 200$300 \mathrm{~g} / \mathrm{cm}^{3}$. The laser energy required for this drive is estimated from simulation [22] to be

$$
E_{\text {laser }}=80\left(\frac{100 \mathrm{gcm}^{-3}}{\rho}\right)^{1.8} \mathrm{~kJ}
$$

yielding the required laser energy of about $50 \mathrm{~kJ}$, while $10-20 \mathrm{~kJ}$ of electron energy needs to be delivered to the spot. Although this idea is potentially capable of reducing the necessary laser energy by almost an order of magnitude or increasing the fusion gain by an order of magnitude at the same laser energy, there remains a considerable uncertainty in the efficiency of energy conversion from short-pulse (10ps) laser to electron beam to the compressed fuel and in the stability and reliability of electron beam. For example, laser and electron beam have to propagate through the over dense plasma, through which filamentation and kinking instabilities are found to arise. In order to cope with this problem, the ignitor laser is further split into the hole boring one and energy deliverer.

We suggest that an alternative method of fast ignition by much shorter-pulse laser may be possible. In this, although the total amount of energy necessary to be delivered is unchanged $(50 \sim 100 \mathrm{~kJ})$, we shorten the pulse length to $10 \mathrm{fs}$ so that the local laser intensity reaches of the order of $10^{25} \mathrm{~W} / \mathrm{cm}^{2}$. Since the resonance frequency reduces inversely proportional to $\sqrt{n_{e}}$, the resonance density becomes in the order of $10^{25} / \mathrm{cm}^{3}$, a very close proximity of the fully compressed fuel. This way we may be avoiding the difficult and long energy transport of electron beam from the density region of $<10^{22} / \mathrm{cm}^{2}$ to $10^{26} / \mathrm{cm}^{3}$. With the recent success of improved target design with a conic aperture for fast ignitor laser beam access [23], our ultrafast approach may be further bolstered as a direct energy delivery vehicle. It remains to be seen, however, how much fraction of the laser energy is consumed due to the pump depletion [24] while it interacts with the surrounding plasma. It also needs to be investigated what kind of electron energy spectrum is generated in the ultra-intense laser beam. The mission of the electron energy conversion in fast ignition is orthogonal to the previous Subsec. 3A of super-high energy electron generation. The production of a small fraction of extremely high-energy electrons is tolerated, as long as the majority of energy is in several $\mathrm{MeV}$ electrons. 


\section{Baryon acceleration}

Many thought it difficult to accelerate protons and heavier particles by light, as massless light propagates at the speed of light, while protons are massive and nonrelativistic - until last year, when the Petawatt Laser experiment [25] and other experiments $[26,27,28]$ showed that protons have been accelerated much beyond a megaelectron-volt. The observed transverse emittance is about $0.5 \mathrm{~mm}$ mrad, while the longitudinal one is about MeV-psec [29]. These early experiments already rival or even surpass those of the conventional ion sources in some of crucial parameters. The main mechanism of laser proton acceleration in the above experiments is due to the space charge set up by energetic electrons that are driven forward away from the back surface of the target slab. The energy of protons is thus dependent on that of electrons. Bulanov and others showed [31] in simulation that at a laser intensity of $I=10^{23} \mathrm{~W} / \mathrm{cm}^{2}$, protons are accelerated beyond a giga-electron-volt. If this process of proton acceleration scales with the intensity (as the electron energy does), we may be able to see $100 \mathrm{GeV}$ protons and $10 \mathrm{TeV}$ at $I=10^{26}$ and $10^{28} \mathrm{~W} / \mathrm{cm}^{2}$, respectively. However, it may also be possible that this process is now due directly to the photon pressure beyond the intensity regime of $I=10^{24} \mathrm{~W} / \mathrm{cm}^{2}$. The energy expected through this mechanism is about the same as that through the space charge mechanism.

It is not clear how much energy will be in protons. In the Petawatt Laser experiment, about $10 \%$ of laser energy $(300 \mathrm{~J}$ ) was converted into proton energy - $30 \mathrm{~J}$ (beyond $1 \mathrm{MeV}$ ) [25]. If we take this conversion efficiency in the extreme relativistic laser intensity, then more than $1 \mathrm{~kJ}$ of proton energy is expected for the case of intensity $10^{26} \mathrm{~W} / \mathrm{cm}^{2}$. If we further take a flat energy spectrum, approximately $10^{11}$ protons are accelerated beyond $10 \mathrm{GeV}$ in this intensity regime. If we can generate the solitary accelerating structure, such energy may exceed $100 \mathrm{GeV}$. If we can converge these in a colliding pair of beams at focus, the luminosity of colliding hadrons is $\mathcal{L}=10^{34} \mathrm{f}$, if we focus on $10 \mathrm{~nm}$. The expected number of nuclear events is on the order of $10^{9}$ per shot.

Such an intense, relativistic, compact proton source has a number of fascinating applications. It may be applicable to ion radiography, fast ignition of fusion, etc., among many others. An additional application is pion (or muon) and neutrino beam generation. With sufficiently relativistic proton energies the emittance of created pions can be sufficiently small. If so, they may be promptly accelerated pions to sufficiently high energies before the space charge effect expands the beam emittance and before they die out. In this the prompt acceleration and its compactness are important, both of which are the forte of the laser accelerations in an application. Protons beyond a certain energy (several hundred $\mathrm{MeV}$ ) in matter induce through the nuclear strong interaction the creation of pions, which in turn decay into muons and neutrinos in a matter of $20 \mathrm{~ns}$, if nonrelativistic, propagating mere $6 \mathrm{~m}$ at most: $\pi \rightarrow \mu+\mathrm{v}$. With the conventional accelerating gradient of, say, $20 \mathrm{MeV} / \mathrm{m}$ over this distance of $6 \mathrm{~m}$, we can increase the pion energy by $120 \mathrm{MeV}$, which will increase the lifetime of pions but not by an order of magnitude. On the other hand, the laser acceleration with its far-greater gradient would increase the energy and lifetime of pions far more than this (and also reduce the emission cone angle). This would contribute to a further smaller emittance, which in turn contributes to higher energy, lower emittance muons and neutrinos. 


\section{Nonlinear QED and horizon physics}

At the intensity $10^{28} \mathrm{~W} / \mathrm{cm}^{2}$, the electric field is only an order of magnitude less than the Schwinger field as discussed below. At this field, fluctuations in vacuum are polarized by laser to yield copious pairs of real electron and positron. In collider physics a similar phenomenon happens when the so-called $\Upsilon$ parameter reaches unity. In reality, even below the Schwinger field, the exponential tail of these fluctuations begins to cause copious pair productions.

Though pair production has been demonstrated at SLAC (E144-experiment) [32] by the interaction of $\gamma$-ray with an intense laser at intensities in the range of $10^{18} \mathrm{~W} / \mathrm{cm}^{2}$, the direct production of pairs by a high-intensity laser from vacuum remains elusive. The rule of thumb for threshold of pair production derives from the simple argument that it is the field necessary for a virtual electron to gain an energy $2 m_{0} c^{2}$ during its lifetime $\delta t$, imposed by the Heisenberg uncertainty principle $\delta t=\hbar / m_{0} c^{2}$, the energy gain length, $c \delta t$ is the Compton length $\pi_{c}$. Hence, the breakdown field $E_{S}$, the Schwinger field, is $E_{S}=m_{0} c^{2} / e \lambda_{c}$ where $\pi_{c}=0.386 \mathrm{pm} E_{S}=2 \cdot 10^{16} \mathrm{~V} / \mathrm{cm}$. (The laser field $E_{S}$ is related to the laser intensity $I_{\ell}$ by $E_{S}^{2}=Z_{0} I_{S}$ where $Z_{0}$ is the vacuum impedance. For $Z_{0}=377 \Omega$, we find a value of $I_{S}=10^{30} \mathrm{~W} / \mathrm{cm}^{2}$ ).

This approach gives an estimate for the threshold for pair creation and do not provide the number of pairs that could be created for a given intensity. The probability of spontaneous production of pair creation per unit time per unit volume by Schwinger [33] is

$$
w=\frac{1}{\pi^{2}} \frac{\alpha}{\delta t} \frac{1}{\pi_{c}^{3}}\left(\frac{E}{E_{S}}\right)^{2} \sum_{n=1}^{\infty} \frac{1}{n^{2}} \exp \left(-n \pi \frac{E_{S}}{E}\right),
$$

where $\alpha$ is the fine structure constant. The number of pairs $N$ for a given laser field is

$$
N=V \tau_{p} w
$$

where $V$ is the focal volume. For $V=10^{-12} \mathrm{~cm}^{3}$ and $\tau_{p}=10 \mathrm{fs}$, we find the generation of $10^{24}$ pairs at the Schwinger intensity $I_{S}$. It is interesting to note that the intensity to create a single pair is still the gargantuan intensity of $10^{27} \mathrm{~W} / \mathrm{cm}^{2}$. However, as indicated earlier (in Subsec. 3E), the presence of matter such as nuclei or electrons reduces this field by a considerable amount.

The interaction of intense laser with high-energy electrons will enhance some of the parameters even further. For example, counterstreaming electron beam and laser can produce copious polarized (high-quality) positrons. This can serve as a polarized positron source for one thing. In an extreme field regime, on the other hand, the laser field is enhanced by the Lorentz factor of the electron beam, so that the effective field from the electron frame may far exceed the Schwinger value. If this field is exceeded by much more than these orders of magnitude, direct production of other particles such as muons out of "vacuum" may be observed.

In addition to the test of nonlinear fields, we are able to explore what may be called 'horizon physics.' According to Einstein's equivalence principle, a particle that is accelerated feels gravity in the opposite direction of the acceleration. The acceleration due to the electric field of the laser at this intensity is huge: $a_{e} \sim 10^{30}$ and $10^{31} \mathrm{~cm} / \mathrm{s}^{2}$, at 
$I=10^{26}$ and $10^{28} \mathrm{~W} / \mathrm{cm}^{2}$, respectively. An observer at rest (or in an inertial frame of reference) sees the horizon at infinity if there is no gravitation. On the other hand, an observer near a black hole sees the horizon at a finite distance where the gravitation diverges. Equivalently, an observer who is being accelerated (feeling immense equivalent gravity) now also sees the horizon at a finite distance. Any particle ("observer" - a wave function) that has a finite extent has one side of its wave function leaking out of the horizon. The Unruh radiation is emitted when this happens [34]. Unruh radiation is a sister to the Hawking radiation [35]. The Unruh temperature

$$
k T_{U}=\frac{\hbar a_{e}}{2 \pi c}
$$

which is about $10^{4} \mathrm{eV}$ and $10^{5} \mathrm{eV}$, for $I=10^{26}$ and $10^{28} \mathrm{~W} / \mathrm{cm}^{2}$, respectively. The radiative power of Unruh radiation increases in proportion to $a_{0}^{3}$ (or $I^{3 / 2}$ ), in contrast to the Larmor radiation power of $a_{0}^{2}$ [36]

$$
P_{U}=\frac{12}{\pi} \frac{r_{e} \hbar}{c} a_{0}^{3} \omega_{0}^{2},
$$

where $r_{e}$ is the electron classical radius. Since in the extreme relativistic regime the radiation is dominated by Larmor radiation, the Unruh signal has to compete with the Larmor with the ratio of powers

$$
\frac{P_{U}}{P_{L}}=\frac{\hbar \omega_{0}}{m_{0} c^{2}} a_{0} .
$$

At this regime, the Unruh is down only by a few orders of magnitude, and it has been suggested to circumvent the noise to observe the Unruh signal by exploiting polarization, etc. [36]. This $P_{U}$ yields $10^{4} \mathrm{eV} / \mathrm{s}$ and $10^{7} \mathrm{eV} / \mathrm{s}$, for $I=10^{26}$ and $10^{28} \mathrm{~W} / \mathrm{cm}^{2}$, respectively.

The shrinkage of the distance to the horizon by the violent acceleration allows us to probe other aspects of gravitational physics. For example, Arkani-Hamed et al. [37] suggested that extra dimensions of the quantum gravity may have manifestations in a relatively low extra dimension $(n)$ in this four-dimensional world. The distance over which this may be manifested is

$$
r_{n} \sim 10^{30 / n-17} \mathrm{~cm} .
$$

The distance to the horizon created by the intense laser acceleration of an electron

$$
d=\frac{c^{2}}{a_{e}}=\frac{\lambda}{2 \pi} \frac{1}{a_{0}},
$$

could exceed the above distance Eq. (19) if $n$ is less than or equal to 4 . 


\section{CONCLUSIONS}

Zetta- and exawatt-lasers will allow us a glimpse into some of the most energetic and enigmatic phenomena of astrophysics such as GRB's, and their associated effects on EHECR, the final energy frontier in the Universe ('Extreme Universe' in the highest energy and from the cosmological distance). We marvel at such fascinating possibilities of exploring fundamental physics that we have just barely scratched the surface so far on this subject. It is also noted that this laser could bring many frontiers of contemporary physics, i.e. particle physics, nuclear physics, gravitational physics, nonlinear field theory, ultrahigh pressure physics, relativistic plasma and atomic physics, fusion science, astrophysics, and cosmology together. Because of such significant potential scientific impacts, though it amounts to nontrivial efforts and developments, it seems worthy of further and more serious consideration of this extreme high-field science. We believe that the contemporary scientific development is timely.

\section{ACKNOWLEDGMENTS}

We appreciated the comment by Profs. N. Fisch, M. Key, and P. Mulser on fast ignition. This work was supported in part by the U.S. Dept. of Energy Contracts DE-FG03-96ER54346, W-7405-Eng. 48, and in part by the National Science

\section{REFERENCES}

1. Strickland, D. , and Mourou, G. A., Opt. Comm., 56, 219 (1985).

2. Mourou, G. A., Barty, C. P. J., and Perry, M. D., Physics Today, January, 1998.

3. Dubeis, A., et al., Opt. Comm., 88, 437 (1992).

4. Morgner, U., Kätner, F. X., Cho, S. H., Chen, Y., Haus, A. H., Fujimoto, J. G., and Ippen, E. P., Opt. Lett., 24, 411 (1999).

5. Druon, F., Cheriaux, G., Faure, J., Nees, J., Nantel, M., Maksimchuk, A., and Mourou, G. A., Opt. Lett., 23, 1043-1045 (1998); Albert, O., Wang, H., Liu, D., Chang, Z., and Mourou, G. A., Opt. Lett., 125, 1125 (2000).

6. Ross, I.N., et al.., Opt. Comm., 144, 125 (1997).

7. Du, D., Liu, X., Korn, G., Squier, J., and Mourou, G. A., Appl. Phys. Lett., 64, 3071 (1994).

8. Stuart, B., Feit, M., Rubenckik, A., Shore, B., and Perry, M., Phys. Rev. Lett., 74, 2248 (1995).

9. Migus, A., Private Communications.

10. M. Felt, Private Communications.

11. Bloembergen, N., Nonlinear Optics, Addison-Wesley, Reading, 1965.

12. Tajima, T., and Dawson, J. M., Phys. Rev. Lett., 43, 267 (1979).

13. Sprangle, P., Tang, C. M., and Esarey, E., IEEE Trans. Plasma Sci., 15, 145 (1987); Barnes, D. C., Kurki-Suonio, T., and Tajima, T., ibid. 15, 154 (1987).

14. Ashour-Abdalla, M., LeBoeuf, J. N.,Tajima, T. , Dawson, J. M., and Kennel,C. F., Phys. Rev. A , 23, 1906 (1981).

15. Tajima, T., Laser Part. Beams, 3, 351 (1985).

16. Scheid, W.and Hora, H., Laser Part. Beams, 7, 315 (1989).

17. Rau, B., Tajima,T., and Hojo, H., Phys. Rev. Lett., 78, 3310 (1997).

18. Lawson, J.D., IEEE Trans. Nucl. Sci., NS-26, 4217 (1979); Woodward, P.M., J. IEE , 93, Part III A, 1554 (1947).

19. Sato, H., and Tati, T., Prog. Theor. Phys., 47, 1788 (1972). 
20. Nakajima, K., this Proceedings, (2001).

21. Tabak, M., et al., Phys. Plasmas, 1, 1626 (1994).

22. Atzeni, Jpn. J. Appl. Phys. (1995).

23. Kodama, R., et al., Nature, 412, 798 (2001)

24. Horton, W., and Tajima, T., Phys. Rev. A, 34, 4110 (1986).

25. Key, M. et al., in First Int'l. Conf. Inertial Fusion Sci. Appl., Bordeaux, France 1999.

26. Maksimchuk, A. et al., Phys. Rev. Lett., 84, 4108 (2000).

27. Clark, E. L., et al., Phys. Rev. Lett., 85, 1654 (2000).

28. Snavely, R.A., et al., Phys. Rev. Lett., (2000).

29. Roth, M., et al., this Proceedings.

30. Esirkepov, T. Zh., Liseikina, T.V., Califano, F., Naumova, N. M., Vshirkov, V. A., Pegoraro, F., Bulanov, S. V., JETP Lett., 70, 82 (1999); also Pukhov, A. et al., in Max-Planck, Y. Ueshima et al., in JAERI, S. Wilks et al., in LLNL, among other groups, have shown similar results.

31. Koga, J. K. et al., this Proceedings.

32. Bula, C., et al., Phys. Rev. Lett., 76, 3116 (1996)

33. Schwinger, J., Phys. Rev., 82, 664 (1951).

34. Unruh, W., Phys. Rev. D., 14, 870 (1976)

35. Hawking, S. W., Nature, London, 248, 30 (1974).

36. Chen, P.S., and Tajima, T., Phys. Rev. Lett., 83, 256 (1999).

37. Arkani-Hamed, N., Dimopoulos, S., and Dvali, G., Phys. Rev. Lett., 84, 586 (2000). 